\title{
Pelvic Congestion Syndrome
}

\author{
Janette D. Durham, MD ${ }^{1}$ Lindsay Machan, MD² \\ ${ }^{1}$ Department of Radiology, University of Colorado Denver, Aurora, \\ Colorado \\ 2 Department of Radiology, University of British Columbia Hospital, \\ Vancouver, BC, Canada \\ Address for correspondence Janette D. Durham, MD, 12401 East 17th \\ Avenue L954, PO Box 6510, Aurora, CO 80045 \\ (e-mail: janette.durham@ucdenver.edu).
}

Semin Intervent Radiol 2013;30:372-380

\begin{abstract}
Patients with pelvic congestion syndrome present with otherwise unexplained chronic pelvic pain that has been present for greater than 6 months, and anatomic findings that include pelvic venous insufficiency and pelvic varicosities. It remains an underdiagnosed explanation for pelvic pain in young, premenopausal, usually multiparous females. Symptoms include noncyclical, positional lower back, pelvic and upper thigh pain, dyspareunia, and prolonged postcoital discomfort. Symptoms worsen throughout the

\section{Keywords}

- pelvic congestion syndrome

- pelvic vein incompetence

- female varicocele

- pelvic pain syndrome

- pelvic vascular congestion

- nutcracker syndrome

- vulvar varices day and are exacerbated by activity or prolonged standing. Examination may reveal ovarian tenderness and unusual varicosities-vulvoperineal, posterior thigh, and gluteal. Diagnosis is suspected by clinical history and imaging that demonstrates pelvic varicosities. Venography is usually necessary to confirm ovarian vein reflux, although transvaginal ultrasound may be useful in documenting this finding. Endovascular therapy has been validated by several large patient series with long-term follow-up using standardized pain assessment surveys. Embolization has been shown to be significantly more effective than surgical therapy in improving symptoms in patients who fail hormonal therapy. Although there has been variation in approaches between investigators, the goal is elimination of ovarian vein reflux with or without direct sclerosis of enlarged pelvic varicosities. Symptom reduction is seen in 70 to $90 \%$ of the treated females despite technical variation.
\end{abstract}

Objectives: Upon completion of this article, the reader will be able to discuss the clinical symptoms and anatomic findings in patients with pelvic congestion syndrome (PCS); evaluate and diagnose a patient with PCS; plan an appropriate treatment strategy in a patient with PCS.

Accreditation: This activity has been planned and implemented in accordance with the Essential Areas and Policies of the Accreditation Council for Continuing Medical Education (ACCME) through the joint sponsorship of Tufts University School of Medicine (TUSM) and Thieme Medical Publishers, New York. TUSM is accredited by the ACCME to provide continuing medical education for physicians.

Credit: Tufts University School of Medicine designates this journal-based CME activity for a maximum of 1 AMA PRA Category 1 Credit $^{\mathrm{TM}}$. Physicians should claim only the credit commensurate with the extent of their participation in the activity.

In the United States, there is a $15 \%$ prevalence of chronic pelvic pain in women 18 to 50 years of age. ${ }^{1}$ The differential diagnosis includes gynecological, genitourinary, gastrointestinal, and musculoskeletal abnormalities of which endometriosis is the most frequently diagnosed condition by laparoscopic examination. ${ }^{2}$ In $60 \%$ of the women presenting with chronic pelvic pain, the cause remains undiscovered. ${ }^{1,2}$

Pelvic congestion syndrome (PCS) can be responsible for chronic pelvic pain when pelvic venous insufficiency (PVI) results in painful pelvic varicosities. Numerous names-pelvic pain syndrome, female varicocele, pelvic vascular congestion (PVC), PCS, and PVI demonstrate a progression in the
Issue Theme Women's Health and Interventional Radiology; Guest Editors, Kimi L. Kondo, DO, and Laura Findeiss, MD, FSIR
Copyright (c) 2013 by Thieme Medical Publishers, Inc., 333 Seventh Avenue, New York, NY 10001, USA. Tel: +1(212) 584-4662. 
understanding of the disease pathophysiology. The condition remains underdiagnosed despite an increasing awareness and acceptance of the condition. Soysal et al found a $31 \%$ incidence of PCS in a population of symptomatic women after evaluation with pelvic examination, laparoscopy, ultrasound, and venography. ${ }^{3}$ In patients in whom intrinsic disease was discovered, the authors found that $12 \%$ also had PCS, while only 1 of 30 asymptomatic patients was found to have PCS.

In a recent study of 273 healthy female kidney donors, the authors found the prevalence of ovarian vein insufficiency to be $9.9 \%$ on preoperative arteriography. ${ }^{4}$ Fifty-nine percent of the patients reported pelvic pain retrospectively, suggesting that a majority of patients with ovarian vein reflux have pain symptoms.

\section{Anatomic Considerations}

An interconnecting plexus of veins drains the bladder, vagina, uterus, and rectum. The lower uterus and vagina drain into the uterine veins and then into branches of the internal iliac veins; the fundus of the uterus drains to either the uterine or the ovarian plexus (utero-ovarian and salpingo ovarian veins) within the broad ligament. The left ovarian plexus drains into left ovarian vein, which empties into left renal vein; the right ovarian plexus drains into the right ovarian vein, which drains into the anterolateral wall of the inferior vena cava (IVC) just below the right renal vein. Rarely, the right ovarian vein drains into the right renal vein. Vulvoperineal veins drain into the internal pudendal vein, then into the inferior gluteal vein, then the external pudendal vein, then into the saphenous vein, or into the circumflex femoral vein, and then into the femoral vein.

\section{Pathophysiology}

Valvular insufficiency, venous obstruction, and hormones all may play a role in the development of "congestion" or sluggish drainage of the utero-ovarian and salpingo ovarian veins. Two anatomic findings define PCS-ovarian vein reflux and pelvic varicosities. Each may be seen without the other or both can be present in asymptomatic patients. Primary pelvic insufficiency includes congenital or acquired ovarian vein incompetence from nonobstructive causes; secondary pelvic insufficiency includes conditions such as nutcracker syndrome (left renal vein compression by the superior mesenteric artery) or May-Thurner syndrome (left iliac vein compression by the right internal iliac artery), where the ovarian or pelvic veins are important collateral pathways to relieve upstream obstruction.

PVI can occur because of absent or incompetent venous valves. Anatomical studies demonstrate valves that prevent ovarian vein reflux at the terminus of the ovarian vein. The numerous pelvic venous plexuses are relatively valve less. Anatomical studies performed by Ahlberg et al demonstrate that ovarian venous valves are absent congenitally in $15 \%$ of the female patients on the left and $6 \%$ on the right, ${ }^{5}$ and valves are incompetent in $41 \%$ of the female patients on the left and $46 \%$ on the right. Incompetent valves and increased venous diameter are found more frequently in multiparous women. Compared with men, the incidence of incompetent valves and increased venous diameter is greater in women; the incidence of absent valves is greater in men. Ovarian veins near their entry into the renal vein measure $3.8 \mathrm{~mm}$ with competent valve and $7.5 \mathrm{~mm}$ with incompetent valve. The upper limit of normal is considered to be $5 \mathrm{~mm}$, the right slightly bigger than the left.

Ovarian varicosities are seen more frequently after pregnancy. ${ }^{6}$ The capacity of pelvic veins may increase 60 -fold over the nonpregnant state, contributing to both venous dilatation and valvular incompetence. There are unique reasons that pelvic veins are particularly liable to become dilated, even without pregnancy. Many pelvic veins are devoid of valves and have weak attachments between the adventitia and supporting connective tissue. ${ }^{7}$ Although this is different from veins elsewhere in the body, the histology of pelvic varicosities is similar to that of varicose veins elsewhere, including fibrosis of the tunica intima and media, muscular hypertrophy, and proliferation of capillary endothelium. The evidence that pelvic varicose veins cause pain is indirect. Symptomatic improvement in patients treated with hormonal manipulation suggests that blocking the direct vasodilator effect of estrogen benefits patients by reducing venous distention. $^{3}$

\section{Diagnosis}

PCS is a clinical syndrome with specific anatomic findingschronic pelvic pain of greater than 6 months duration secondary to PVI and associated pelvic venous distention. ${ }^{8}$ Patients are most often multiparous, premenopausal females, ranging in age from 20 to 40 years. Symptoms include noncyclical, positional lower back, pelvic, and upper thigh pain. Pain is exacerbated before or during menses and may be associated with dyspareunia and prolonged postcoital discomfort. Symptoms are generally most severe at the end of the day, exacerbated by standing or heavy activity, and are diminished with supine positioning. Other complaints may include lumbosacral neuropathy, urinary frequency, and generalized lethargy.

Pelvic examination may demonstrate cervical motion and ovarian point tenderness. The combination of postcoital ache and ovarian point tenderness is reported to be $94 \%$ sensitive and $77 \%$ specific for PVI when confirmed by venography. ${ }^{9}$ Vulvoperineal varicosities can be found in 4 to $8.6 \%$ of the patients with lower extremity venous insufficiency and may accompany ovarian vein insufficiency. ${ }^{10}$ These varices can extend over the buttock and posteriomedial thigh and communicate with both greater and lesser saphenous veins. They most commonly manifest during pregnancy and regress postpartum.

Referral patterns dictate to some extent the diversity of patients undergoing evaluation of PCS. The classic findings described above are often those of a patient referred by a gynecologist in pursuit of an explanation for chronic pelvic pain. Patients are also referred for evaluation of incidentally discovered pelvic varicosities diagnosed by imaging examination for other indications. These patients may be entirely 
asymptomatic, in which case therapy is not indicted. Pelvic varices may be a result of intrinsic pelvic pathology, caused by local obstruction. Therapy is directed toward treatment of the underlying pathology. Vein specialists may send patients for evaluation of PVI who have atypical or no pain symptoms, but a clinical presentation including hemorrhoids or incidentally discovered vulvoperineal or posterior upper thigh varices. PVI may also explain some cases of lower extremity venous insufficiency treatment failure. ${ }^{11}$ Patients undergoing evaluation for PCS and PVI therefore fall into the following three groups: those with incidentally found pelvic varices, those with unusual vulvar or upper thigh varices that complicate lower extremity insufficiency with or without pelvic pain, and patients with painful pelvic varicosities secondary to PVI who in fact have PCS.

\section{Imaging Evaluation}

The evaluation of chronic pelvic pain starts with the exclusion of the most common causes of intrinsic pelvic pathology, including endometriosis, pelvic inflammatory disease, postoperative adhesions, and uterine pathology including adenomyosis or leiomyoma. ${ }^{3}$ Magnetic resonance imaging (MRI), laparoscopy, transabdominal ultrasound (US), and computed tomographic (CT) imaging can be used to exclude intrinsic disease, but all of these diagnostic studies are limited in their sensitivity for pelvic varices, in part because of supine positioning, compression resulting from $\mathrm{CO}_{2}$ insufflation during laparoscopy, and perhaps normal hydration and hormonal variation. The sensitivity of these examinations for PCS is low $-59,40,20$, and $13 \%$ for MRI, laparoscopy, US, and CT, respectively. $^{12}$

In experienced hands, transabdominal combined with transvaginal ultrasound, performed in a supine or semiupright position has been found to be useful in demonstrating both pelvic varices and pathologic ovarian venous reflux. It is a practical first step in evaluation for PCS that can exclude intrinsic pelvic conditions, demonstrate pelvic varicosities, and suggest ovarian vein insufficiency. Sonographic findings include enlarged ovarian veins greater than $6 \mathrm{~mm}$ in diameter with reversed blood flow, presence of pelvic varicocele ( $>$ $5 \mathrm{~mm}$ ), and dilated ( $>5 \mathrm{~mm}$ ) arcuate veins crossing the uterine myometrium between pelvic varicoceles. ${ }^{13}$ Response of pelvic varices to Valsalva maneuvers can be variable because of their intraperitoneal location. An unexplained increased incidence of polycystic ovaries is detected in this population who do not have amenorrhea. This ovarian ultrasound examination is an easy addition to Doppler US lower extremity examination, when pelvic reflux is suggested or vulvoperineal varicosities are identified.

Despite negative findings for PVI on any of the above studies, venography is indicated in the appropriate clinical setting when a suspicion for PCS exists. It can be performed for confirmation of the condition, at the same time that treatment is planned. Left renal, bilateral gonadal, iliac, and internal iliac venography is performed. Pressures across the renal and left common iliac vein are obtained whenever an abnormality is detected by imaging. Venographic findings include renal vein reflux into dilated ovarian veins ( $>5 \mathrm{~mm}$ ), stagnation of contrast in the pelvic veins, contralateral reflux across the midline, and demonstration of vulvoperineal or thigh varices. ${ }^{14}$

\section{Treatment}

Successful descriptions of retroperitoneal surgical ovarian vein ligation to treat PCS preceded the first case report by Edwards et al of bilateral ovarian vein embolization with steel endovascular coils. ${ }^{15,16}$ Since his description, coil embolization of the ovarian vein, unilaterally or bilaterally, has been the most common approach to eradicate ovarian vein reflux. Multiple reports demonstrate the success of this approach with favorable outcomes, including partial or significant relief of symptoms in 70 to $100 \%$ of the patients. The left ovarian vein has been the most commonly occluded vein in most reported series. Kwon et al reported a series of 67 patients, of whom 64 patients had left ovarian vein occlusion, 1 right ovarian vein occlusion, and 2 bilateral ovarian vein occlusion. ${ }^{17}$ He demonstrated improvement in $82 \%$ with coil embolization alone. The largest series of patients with PCS treated with endovascular therapy to date, published by Laborda et al, includes 202 patients with pelvic pain selected from a population of patients with lower extremity venous insufficiency. ${ }^{11}$ These authors utilized coil occlusion alone, with the intention to treat all refluxing veins including both ovarian and appropriate branches of both iliac veins. Nearly all patients had at least three of these four veins treated. Clinical benefit was seen in $94 \%$ of the patients utilizing a visual analog scale (VAS) pain questionnaire. After treatment the clinical response continued to improve for most of the first year. Lasting and significant benefit was documented with VAS pain scores decreasing from 7.3 to 0.8 (10-point scale) in the $89 \%$ of the patients who were followed for 5 years. These authors reported a $12.5 \%$ recurrence rate of lower extremity varices in this population of patients who had both lower extremity insufficiency and PCS at presentation.

Extension of the focus of therapy, from ovarian vein occlusion to sclerosis of the abnormal pelvic veins directly, was introduced in a publication of 56 patients by Venbrux et al in $2002 .{ }^{18}$ These authors injected $5 \%$ sodium morrhuate mixed with gelfoam into the pelvic veins from the ovarian vein before its occlusion with stainless steel or platinum coils. In a staged fashion, at a separate procedure, appropriate branches of the internal iliac vein were then embolized in the majority of patients by injecting sclerosant through an occlusion balloon. Significant and partial response was seen in $96 \%$ of the subjects in this report. Pain assessed by a VAS questionnaire showed a decrease in pain level from 7.8 to 2.7 at 12 months. Kim et al expanded this report in 2006, updating the series to include 127 patients. ${ }^{12}$ Mean follow-up was 45 months with $83 \%$ of patients experiencing durable clinical improvement. The recurrence rate of pelvic pain was $5 \%$.

Multiple sclerosants have been utilized in combination with ovarian vein mechanical occlusion. Gandini et al in 2008 reported the use of $3 \%$ sodium tetradecyl sulfate (STS) foam$2 \mathrm{~mL}$ of STS mixed with $8 \mathrm{~mL}$ of air-to treat 38 patients with 
PVI. ${ }^{19}$ These authors injected foam until pelvic venous stasis was demonstrated and did not subsequently place coils. They noted an improvement in $100 \%$ of treated patients. Injection volumes were typically $30 \mathrm{~mL}$ on the left and $20 \mathrm{~mL}$ on the right. The right side was treated only when varices did not cross the midline from left to right. Procedural cost and radiation time appear to be favorable with such a sclerosant-only approach.

This series was also notable for antecubital access. The transjugular or antecubital approaches allow more straightline access to the pelvic veins and right ovarian cannulation can be performed without a catheter exchange. However, many experienced practitioners utilize the femoral approach with high technical success rates.

A tilting table can be helpful but is not essential when performing venography. If the choice is between a nontilting angiographic table and a tilting table without rapid-sequence image acquisition, the latter is recommended. The volume of sclerosant required typically ranges from 2.5 to $12.5 \mathrm{cc}$ per ovarian vein.

Controversy remains concerning treatment of upstream obstruction when PVI is secondary to renal vein compression (nutcracker syndrome) or reflux into the iliac veins caused by compression of the left iliac vein (May-Thurner syndrome). Failure to address obstruction leads to failed results or partial responses that progress to failure. Failure to detect an upstream obstruction may help explain the fact that a small number of patients are reported to be worse after ovarian vein occlusion. Occluding dominant collaterals might be expected to worsen the condition.

There is a large experience with iliac venous stent placement to treat patients with iliac vein thrombosis that would suggest this approach would be well tolerated in PCS patients presenting with May-Thurner syndrome but without venous thrombosis. There is less experience with endovascular treatment of renal vein compression in patients with nutcracker syndrome. Early experiences with endovascular stent placement to treat renal vein compression demonstrated an infrequent but significant incidence of stent migration (often to the right atrium) that necessitated surgical removal. This has led to a bias favoring more invasive surgical approaches, although the data to support surgery are limited and comprised of small retrospective series and case reports. ${ }^{20}$ In the meantime, stent technology has progressed to provide long (4-6 cm), large $(12-16 \mathrm{~cm})$ self-expanding stents that are better configured for treatment of a renal vein compression near the confluence with the IVC. Although a recommendation to treat renal vein compression with large self-expanding stents is premature, the clinical experience is growing. ${ }^{21}$

Medical ovarian suppression with medroxyprogesterone or goserelin, or surgical ovarian suppression with bilateral salpingo-oophorectomy, has also been used to treat PCS. The limited duration of response to medical hormonal suppression and the morbidity of major surgery to remove a normal uterus and both ovaries favorably positions endovascular approaches. A prospective comparison of percutaneous endovascular versus surgical therapies has been published. ${ }^{22}$ One hundred sixty-four women with chronic pelvic pain were diagnosed with PCS after laparoscopy and venography. After a nonresponse to a 4 to 6 month trial of hormonal therapy, 118 women were enrolled. Randomization to ovarian vein embolization, hysterectomy with bilateral oophorectomy, and hysterectomy with unilateral oophorectomy was performed. Pain assessment scores using a VAS pain scale and standardized stress questionnaire were compared at presentation, and 3,6 , and 12 months following treatment. Embolization was found to be significantly more effective at reducing pelvic pain compared with surgery, except for those patients with the highest stress scores, in whom the benefit over surgery was lost at 1 year. Embolization was completed with coils alone-unilateral left ovarian in 90 , right in 8 , and bilateral in 8 patients. In the surgical group, nine patients were excluded because of the discovery of other pelvic pathology and three patients for failure to operate on the involved ovary. Single oophorectomy had the least effective results, supporting the need for complete hormonal suppression in order for surgery to be beneficial. There may be a role for surgery in patients who fail embolization, but surgery in this setting can fail to result in complete responses, emphasizing again the complex nature of this disease.

\section{Complications}

Major complications are rare. Venous access site complications have been reported as frequently as $3 \%$ in one series ${ }^{11}$; large venous collaterals can surround the femoral vein, and US should be used to guide access and to avoid their inadvertent puncture. Coil migration from the upper ovarian vein into the renal vein or from large iliac veins into the pulmonary arteries seems to occur not infrequently, with most authors who describe iliac vein embolization reporting this complication in 3 to $4 \%$ of the patients. ${ }^{12,17,22}$ Mild to moderate postembolization pelvic and flank pain is common and controlled with anti-inflammatory medications. Pain seems to be worse with the use of sclerosants. Minor complications include spasm of the ovarian vein preventing successful embolization, inability to catheterize an intended target, and perforation of a vein that then limits the safety of sclerosis.

\section{Preprocedure Care}

Timing of the procedure in relation to menstrual or pain cycle is unimportant. The patient should be restricted to clear fluids after midnight for a morning appointment, and clear fluids after breakfast for an afternoon appointment. Admission to a day care bed should be considered as considerable sedation may be required. As important as technical excellence during the procedure is the manner in which the interventional team interacts with these patients and their clinician. These patients may require more time before and after the procedure and more analgesia during it.

\section{Postprocedure Care and Follow-Up}

Following the procedure, the patient is observed for several hours to permit hemostasis at the puncture site. Once 
ambulatory, they are instructed to avoid heavy lifting or exertion more intense than walking for 3 to 7 days postdischarge. Anti-inflammatory medications are used to control postprocedural pain; oral narcotics are rarely required. The first menstrual period after embolization is often unusually heavy. Patients should be warned and reassured that this is almost invariably transient.

Patients should be re-evaluated at 3 months for clinical response, at which time a transvaginal ultrasound to assess ovarian vein or pelvic vein reflux can be performed, providing an objective treatment assessment. Ovarian hormone levels following the procedure have been shown not to be affected. ${ }^{12}$ Whether or not hormonal suppression might improve results has not been studied. Counseling to mitigate the emotional stress that frequently accompanies chronic pain conditions or to address narcotic dependence may be indicated.

Patients may describe persistent symptoms at 3 months. If the presentation was chronic pelvic pain, careful questioning may reveal that symptoms have improved but have not completely resolved. It is important to temper patient expectations as many patients will have less frequent, less severe pain rather than complete symptom eradication as their ultimate outcome. Improvement of chronic pelvic pain may be delayed for more than 6 months posttreatment, particularly when severe at presentation. If the patient has persistent, unimproved pain at 6 months, repeat venography may be indicated to evaluate for a recanalized ovarian vein, a missed cause for reflux, continued filling of pelvic varicosities, or an undiagnosed outflow obstruction. Consideration should be given to the addition of other treatments such as physical therapy, neurostimulation, or trigger point injections.

If the presentation was vulvar or lower extremity varicosities, most often there will be minimal change at follow-up. The principle role of ovarian vein embolization is elimination of the highest point of reflux. Ablation or resection of vulvoperineal or leg varicosities that have not resolved can be performed with lesser chance of recurrence.

\section{Case 1}

A 43-year-old gravida 3, para 3, physician's wife presented with a 7-year history of pelvic pain that had worsened since her last pregnancy 4 years earlier. During that pregnancy, she had developed large left-sided vulvar varices which had resolved postdelivery, and hemorrhoids, which had not. The pain was a dull generalized ache, worse with exercise, at the end of the day, and excruciating for 2 to 3 days before her period. On her worst days, narcotic analgesics were required and she was unable to work or perform most normal activities of daily living. She was avoiding sexual activity because of severe cramping that occurred after intercourse and she was concerned this was affecting her marriage. She noted progressive bilateral spider veins but denied lower extremity varicosities. Her mother and grandmother both had "terrible" varicose veins and suffered from severe pelvic pain, in each case partially im- proved by hysterectomy. She was seen by a gynecologist, who performed a normal laparoscopy. Previous treatments included naturopathy, psychotherapy, hormone suppression, physiotherapy, and pressure point injections, all of which gave transient or no relief.

After reading about PCS online, she was told by her gynecologist that "it was nonsense." She consulted a second gynecologist who agreed with the first and complained to her husband. After a further year of pain, she contacted the radiology department directly, asking for a consultation. Transvaginal ultrasound demonstrated dilated ovarian veins with brisk accentuation of blood flow with Valsalva maneuver. ( - Fig. 1A, B)

\section{Approach}

Before venography the patient was sedated. Despite being petite, a total of $5 \mathrm{mg}$ of versed and $200 \mu \mathrm{g}$ of fentanyl were given during the procedure. After ultrasound-guided puncture, a sheath was introduced into the right internal jugular vein. A multipurpose catheter was directed into the left renal vein and a diagnostic renal venogram performed during Valsalva maneuver (-Fig. 1C). Selective injection of the left ovarian vein was performed demonstrating retrograde flow within the ovarian vein and opacification of paraovarian varicosities (-Fig. 1D). The catheter was advanced into the distal left ovarian vein and forceful injection performed to identify all collateral channels. In total, 7 cc of STS (Tromboject, Omega Laboratories, Montreal, Canada), each 2 cc opacified with 0.5 cc of nonionic contrast, was infused as the patient performed a Valsalva maneuver until there was stasis of flow (-Fig. 1E). The more proximal vein was occluded with 38-8-10 MREye coils (Cook, Inc., Bloomington, IN) "layed out" rather than tightly packed (-Fig. 1F). This method of coil deployment involves holding the coil pusher in place as the catheter is withdrawn, thus the coil is uncovered and deployed in elongated form.

The same multipurpose shape catheter was directed into the right renal vein to define the right renal vein origin. As is typical, the right ovarian vein arose from the vena cava immediately caudal and ventral to the renal vein orifice. Selective right ovarian venogram demonstrated retrograde flow and smaller varices, partially occluded by the sclerosant infused from the left ( - Fig. 1G). Embolization was performed in the same fashion as described for the left. Bilateral internal iliac venograms were the performed during Valsalva maneuver and using the same multipurpose catheter. As these were normal, no further embolization was performed (the authors do not embolize the internal iliac veins unless varicosities are demonstrated).

Three months postembolization, the patient was delighted with the outcome. Most days she noted intermittent mild pelvic ache requiring no treatment. On her worst days, nonsteroidal anti-inflammatory agents were adequate for pain control. Her dyspareunia had not resolved. Transvaginal ultrasound showed residual paraovarian veins, smaller in comparison to pre-embolization and with normal Valsalva accentuation of blood flow (-Fig. 1H). 
A
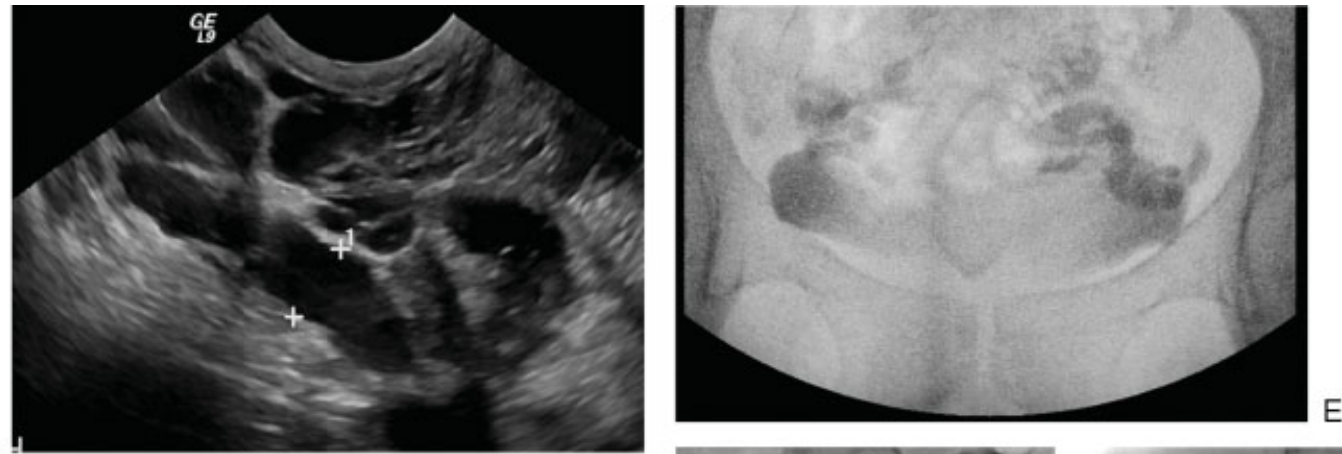

B
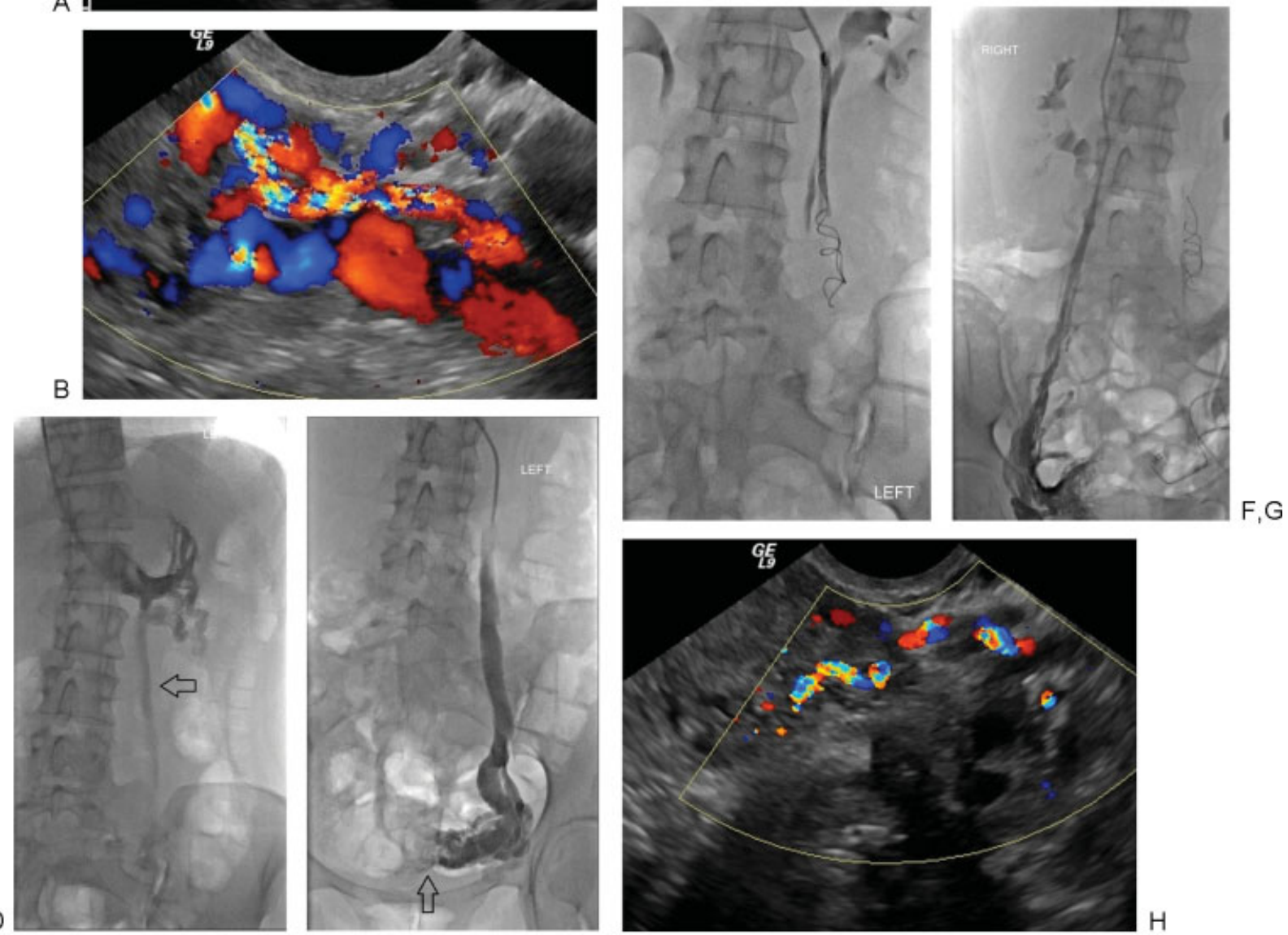

Figure 1 (A and B) Transvaginal ultrasound demonstrating dilated paraovarian (uterineovarian and salpingo ovarian) veins. Color Doppler examination revealing augmentation of blood flow with Valsalva maneuver. (C) Renal venography demonstrates ovarian vein insufficiency during Valsalva. The ovarian vein is dilated (arrow). (D) Selective ovarian venography demonstrates dilated paraovarian veins with faint filling of uterine veins crossing the midline (arrow). (E) After sodium tetradecyl sulfate foam sclerotherapy, there is stagnant flow in bilateral paraovarian veins. (F) Layed-out coils in the left ovarian vein. (G) Selective right ovarian venography demonstrates dilated right paraovarian veins prior to sclerotherapy and coil occlusion of the left ovarian vein. $(\mathrm{H})$ Transvaginal ultrasound following treatment demonstrating markedly diminished flow in the paraovarian veins (compare with $\mathbf{-}$ Fig. 1B).

\section{Discussion}

This patient's presentation has many features typical of PCS. The syndrome is poorly understood and many physicians do not accept its existence. Despite having symptoms severe enough to affect her work and marriage, she had to go to extraordinary lengths just to be evaluated, straining her relationship with her gynecologist. For an interventional radiologist, the procedure is simple; obtaining referrals and learning the complexities of managing patients with a chronic pain syndrome are more difficult.

\section{Case 2}

A 31-year-old thin, premenopausal, nulliparous woman was referred for evaluation of pelvic pain that had worsened over the last 2 years, specifically in left inguinal area. She also had left flank pain and a diagnosis of nutcracker syndrome. She did not have hematuria. Her pain was worse late in the day and was often excruciating. She avoided strenuous activity that worsened her pain. Her pain was worse with menses and ovulation, and she felt exhausted. 
She also had bothersome hemorrhoids and urinary frequency, as well as postcoital pain. Physical examination did not reveal lower extremity or vulvoperineal varicosities. A CT demonstrated renal vein compression and an enlarged left gonadal vein (-Fig. 2A), as well as prominent bilateral ovarian and uterine veins.

\section{Approach}

Left renal venography demonstrated reflux into an enlarged ovarian vein (-Fig. 2B). There was dense staining of the uterine veins and cross-filling to the right internal iliac vein (-Fig. 2C). The origin of the right ovarian vein was not found. She did not demonstrate iliac vein reflux. The left renal vein was compressed, although flow was antegrade into the IVC.
The ovarian vein was catheterized with a microcatheter and infused with $3 \%$ STS foam until stasis, and coils were deployed in the ovarian vein ( - Fig. 2D).

At 1-month follow-up, her pelvic symptoms had resolved, but her left flank pain persisted. By 4 months, this had not changed. Surgical renal vein transposition and endovascular stent placement were discussed, and she elected the latter. On follow-up venography, she was found to have a recanalized upstream gonadal vein filling from an enlarged ovarian vein collateral arising close to the drainage of the previously embolized ovarian vein into the renal vein, and running parallel to the previously occluded channel (-Fig. 2E, F). This was catheterized and embolized, following reinjection of her pelvic varices with $3 \%$ of STS foam. Following this, a
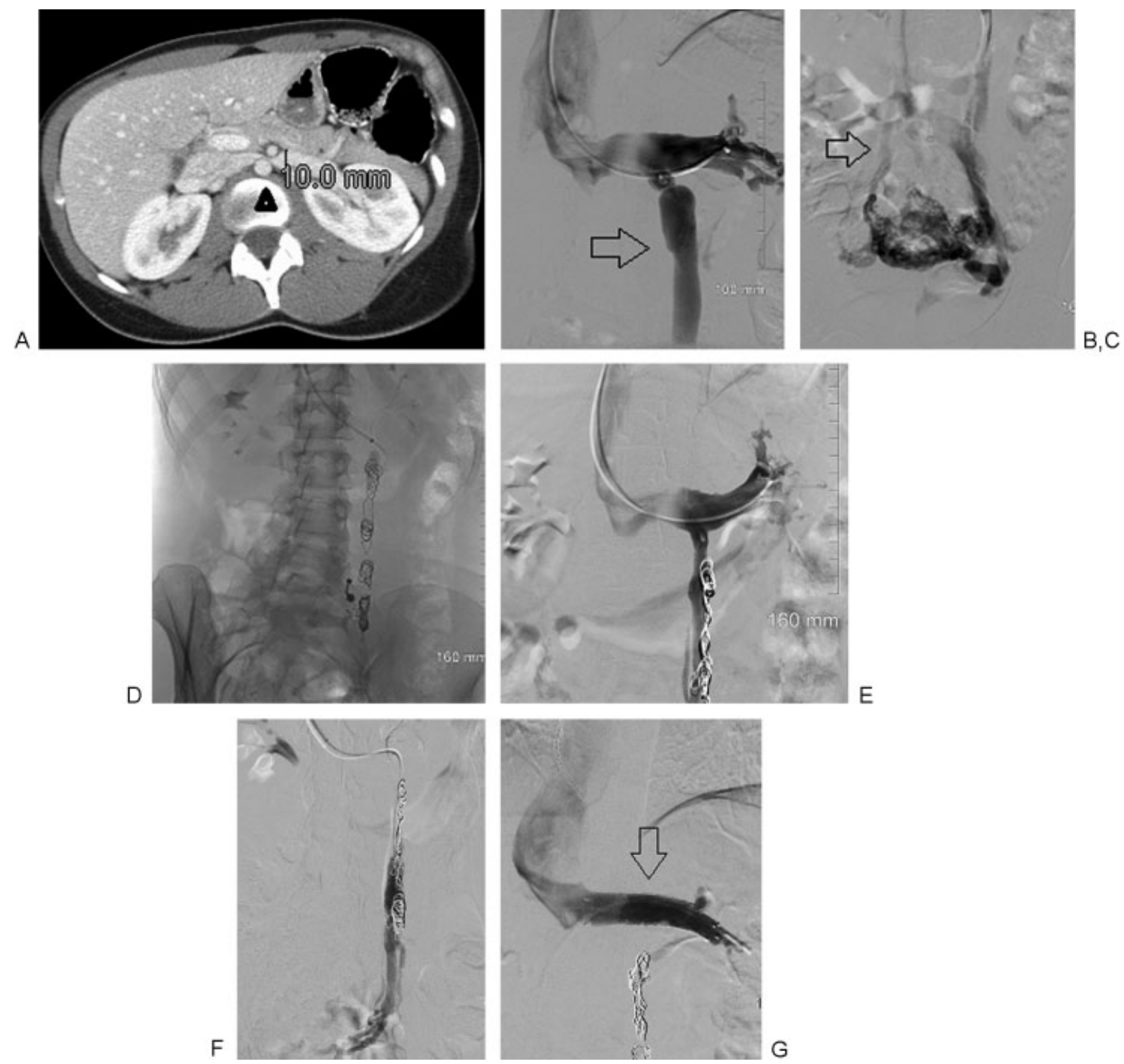

Figure 2 (A) Computed tomographic demonstration of renal vein compression (arrowhead) between the anterior superior mesenteric artery and the aorta, with upstream renal vein dilation. (B) Renal venography demonstrating ovarian vein reflux into a dilated left ovarian vein (arrow). (C) Selective ovarian venography demonstrating large paraovarian veins. Dilated uterine veins cross the midline to fill right paraovarian veins that drain into the right internal iliac vein (arrow). (D) Coil occlusion of the left ovarian vein after sclerotherapy of the paraovarian veins. (E and F) Renal venography at 4 months following the original procedure demonstrates reflux into a dilated vein that runs parallel to the coil pack and fills left paraovarian veins. (G) Renal venography following sclerotherapy of the recanalized ovarian vein and coil occlusion. A $14 \mathrm{~mm}$ by $4 \mathrm{~cm}$ selfexpanding stent has been placed across the renal compression (arrow). No further reflux is demonstrated. 


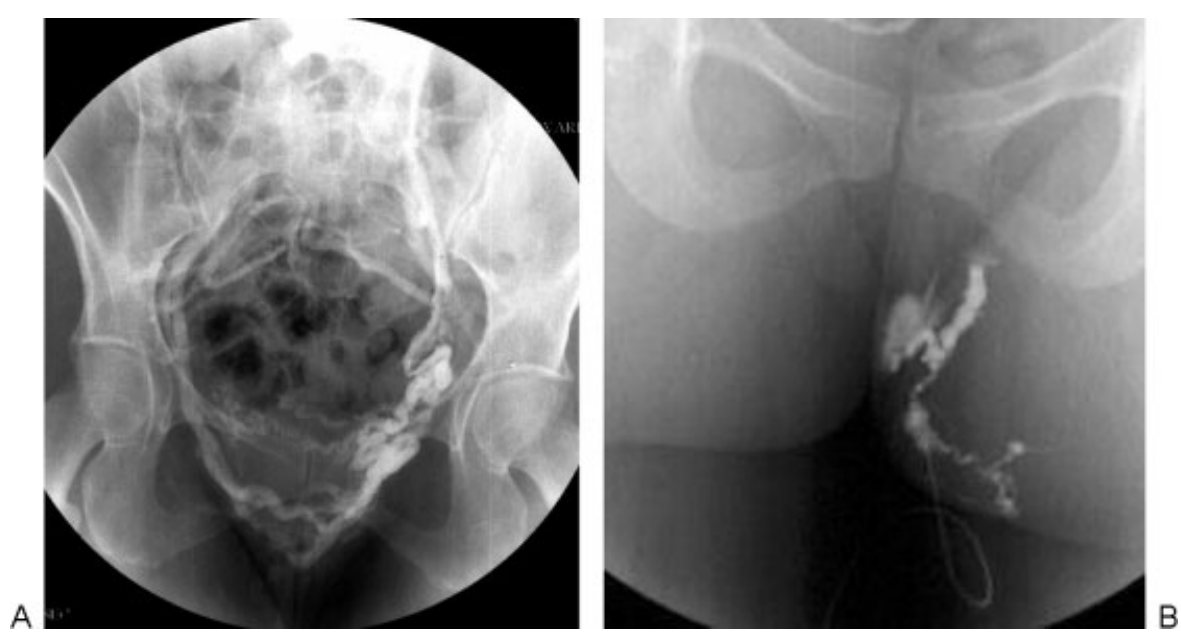

Figure 3 (A) Selective left ovarian venography fills paraovarian and vulvar varices that cross the midline and drain through the right internal iliac vein. (B) Direct puncture and injection of a left vulvar varicosity to plan sclerotherapy injection volume.

$14 \mathrm{~mm}$ to $4 \mathrm{~cm}$ self-expanding stent was placed in the renal vein (-Fig. 2G). Postprocedure, Flank pain developed post procedure and required 48 hours of oral narcotics.

\section{Discussion}

Although PCS is most common in multiparous females, prior pregnancy is not a requirement for this condition. Kim et al reported an unusually high incidence of nulliparous females (63\%) in his series. ${ }^{12}$ Perhaps secondary causes of PVI will be found to occur more frequently in this population of patients. This case demonstrates the need to relieve downstream obstruction when PVI is found to be secondary to another underlying cause. New collateral channels tend to develop when hemodynamic obstruction is not relieved. Flank pain appears distinct from pelvic pain in patients with nutcracker syndrome.

\section{Case 3}

A 45-year-old gravida 4, para 2, woman with recurrent lower extremity varices was referred by her vascular surgeon for evaluation and treatment of vulvar varices. The year prior, bilateral greater saphenous vein (GSV) stripping and sclerotherapy were performed for cosmetic treatment of lower extremity varicose veins. The varicose veins of the right leg had recurred; veins on the left had never improved. Apart from irritation from the vulvar varices, none of the veins were symptomatic and the patient denied pelvic pain. Clinical examination revealed extensive varicose veins in the greater saphenous distribution bilaterally, and left vulvar varicosities. Transvaginal ultrasound demonstrated dilated paraovarian veins with abnormal Valsalva flow accentuation, worse on the left. On duplex ultrasound of the right leg, the GSV was not visible, but there was Grade III reflux in out-of-compartment parallel venous channels. On the left, a leash of collaterals reconstituted the GSV approximately $5 \mathrm{~cm}$ distal to the saphenofemoral junction, resulting in Grade IV reflux extending to below the knee.

\section{Approach}

Marked left ovarian vein reflux was seen on left renal venography. Selective left ovarian vein injection demonstrated opacification of bilateral vulvar varicosities ( - Fig. 3A). The left ovarian vein was embolized using STS and coils. The right ovarian vein, both internal iliac veins, and left common iliac venography were normal.

The patient had an uneventful recovery. At 3 months, her vulvar and varicose veins were unchanged. She was placed on a tilt table, elevated to 45 degrees, and was asked to Valsalva before puncture to dilate the veins. Using local anesthesia only and visual guidance, the largest labial varix on each side was cannulated with a 25 gauge butterfly needle, and contrast injected to estimate the volume of required sclerosant (-Fig. 3B). Approximately, 2.5 cc of $0.5 \%$ STS foam was injected on the left and $1.5 \mathrm{cc}$ on the right. After 1 minute of manual compression, the patient put on compression shorts, which she was advised to wear for 1 day. Subsequently, ultrasound-guided foam sclerotherapy was performed on the right leg varicosities and endovenous laser therapy on the left GSV, both in a vein clinic with good effect.

\section{Discussion}

Ovarian and pelvic vein embolization has become a significant part of the management of lower extremity venous disease. Even when connections with the clinically evident veins are not demonstrated, as was the case with the varicosities in each greater saphenous distribution in this patient, it is important to treat the highest point of reflux. In most cases, even when there is marked ovarian vein reflux, the vulvar, gluteal, or lower extremity varicosities are not opacified.

\section{Conclusion}

PCS is increasingly being diagnosed both clinically and by imaging in patients with pelvic pain. Referral patterns are changing to include women being screened for lower extremity venous insufficiency. This focus is increasing the overall awareness of the condition and increasing referrals. When 
ovarian venous insufficiency is the cause of chronic pelvic pain secondary to pelvic varices, endovascular therapy is indicated to eliminate ovarian reflux. If the etiology is secondary to upstream obstruction, that obstruction needs to be ameliorated. Improvement in pain symptoms should be expected in 80 to $90 \%$ of the patients, with significant improvement in $60 \%$ of the patients. Treatment appears durable at 5 year follow-up.

There remains considerable variation in the endovascular approach, and the optimum approach remains to be elucidated. Improved reporting with standardized diagnostic tools that include pain assessment and quality of life surveys, and long-term result reporting with objective imaging and clinical parameters, have increased acceptance of the procedure by insurance companies. Societal guidelines have also been important in helping to build consensus for endovascular treatment. The Society of Vascular Surgery has endorsed with a $2 \mathrm{~B}$ recommendation the endovascular treatment of PCS in their published practice guidelines for treatment of chronic venous disease. ${ }^{23}$

\section{References}

1 Mathias SD, Kuppermann M, Liberman RF, Lipschutz RC, Steege JF. Chronic pelvic pain: prevalence, health-related quality of life, and economic correlates. Obstet Gynecol 1996;87(3):321-327

2 Farquhar CM, Rogers V, Franks S, Pearce S, Wadsworth J, Beard RW. A randomized controlled trial of medroxyprogesterone acetate and psychotherapy for the treatment of pelvic congestion. $\mathrm{Br} \mathrm{J}$ Obstet Gynaecol 1989;96(10):1153-1162

3 Soysal ME, Soysal S, Vicdan K, Ozer S. A randomized controlled trial of goserelin and medroxyprogesterone acetate in the treatment of pelvic congestion. Hum Reprod 2001;16(5):931-939

4 Belenky A, Bartal G, Atar E, Cohen M, Bachar GN. Ovarian varices in healthy female kidney donors: incidence, morbidity, and clinical outcome. AJR Am J Roentgenol 2002;179(3):625-627

5 Ahlberg NE, Bartley O, Chidekel N. Right and left gonadal veins. An anatomical and statistical study. Acta Radiol Diagn (Stockh) 1966; 4(6):593-601

6 Hodgkinson CP. Physiology of the ovarian veins during pregnancy. Obstet Gynecol 1953;1(1):26-37

7 Viala JL, Flandre O, Girardot B, Maamer M. [Histology of the pelvic vein. Initial approach]. Phlebologie 1991;44(2):369-372, discussion 373

8 Black CM, Thorpe K, Venrbux A, et al. Research reporting standards for endovascular treatment of pelvic venous insufficiency. J Vasc Interv Radiol 2010;21(6):796-803
9 Beard RW, Reginald PW, Wadsworth J. Clinical features of women with chronic lower abdominal pain and pelvic congestion. $\mathrm{Br} \mathrm{J}$ Obstet Gynaecol 1988;95(2):153-161

10 Jung SC, Lee W, Chung JW, et al. Unusual causes of varicose veins in the lower extremities: CT venographic and Doppler US findings. Radiographics 2009;29(2):525-536

11 Laborda A, Medrano J, de Blas I, Urtiaga I, Carnevale FC, de Gregorio MA. Endovascular treatment of pelvic congestion syndrome: visual analog scale (VAS) long-term follow-up clinical evaluation in 202 patients. Cardiovasc Intervent Radiol 2013;36(4): 1006-1014

12 Kim HS, Malhotra AD, Rowe PC, Lee JM, Venbrux AC. Embolotherapy for pelvic congestion syndrome: long-term results. J Vasc Interv Radiol 2006;17(2, Pt 1):289-297

13 Park SJ, Lim JW, Ko YT, et al. Diagnosis of pelvic congestion syndrome using transabdominal and transvaginal sonography. AJR Am J Roentgenol 2004;182(3):683-688

14 Beard RW, Highman JH, Pearce S, Reginald PW. Diagnosis of pelvic varicosities in women with chronic pelvic pain. Lancet 1984; 2(8409):946-949

15 Rundqvist E, Sandholm LE, Larsson G. Treatment of pelvic varicosities causing lower abdominal pain with extraperitoneal resection of the left ovarian vein. Ann Chir Gynaecol 1984;73(6): 339-341

16 Edwards RD, Robertson IR, MacLean AB, Hemingway AP. Case report: pelvic pain syndrome-successful treatment of a case by ovarian vein embolization. Clin Radiol 1993;47(6):429-431

17 Kwon SH, Oh JH, Ko KR, Park HC, Huh JY. Transcatheter ovarian vein embolization using coils for the treatment of pelvic congestion syndrome. Cardiovasc Intervent Radiol 2007;30(4):655-661

18 Venbrux AC, Chang AH, Kim HS, et al. Pelvic congestion syndrome (pelvic venous incompetence): impact of ovarian and internal iliac vein embolotherapy on menstrual cycle and chronic pelvic pain. J Vasc Interv Radiol 2002;13(2, Pt 1):171-178

19 Gandini R, Chiocchi M, Konda D, Pampana E, Fabiano S, Simonetti G. Transcatheter foam sclerotherapy of symptomatic female varicocele with sodium-tetradecyl-sulfate foam. Cardiovasc Intervent Radiol 2008;31(4):778-784

20 Venkatachalam S, Bumpus K, Kapadia SR, Gray B, Lyden S, Shishehbor MH. The nutcracker syndrome. Ann Vasc Surg 2011;25(8): 1154-1164

21 Zhang H, Li M, Jin W, San P, Xu P, Pan S. The left renal entrapment syndrome: diagnosis and treatment. Ann Vasc Surg 2007;21(2): 198-203

22 Chung $\mathrm{MH}$, Huh CY. Comparison of treatments for pelvic congestion syndrome. Tohoku J Exp Med 2003;201(3):131-138

23 Gloviczki P, Comerota AJ, Dalsing MC, et al; Society for Vascular Surgery ; American Venous Forum. The care of patients with varicose veins and associated chronic venous diseases: clinical practice guidelines of the Society for Vascular Surgery and the American Venous Forum. J Vasc Surg 2011;53(5, Suppl): 2S-48S 